\title{
Design Aspects of a Broadband Beam-Reconfigurable Leaky-Wave Antenna
}

\author{
E. Abdo-Sánchez, D. Palacios, C. Frías, F.Y. Ng-Molina, T.M. Martín-Guerrero, and C. Camacho-Peñalosa \\ Dpto. Ingeniería de Comunicaciones, E.T.S.I. Telecomunicación, Universidad de Málaga, Andalucía Tech, \\ E-29071 Málaga, Spain. Email: elenaabdo@ic.uma.es
}

\begin{abstract}
Design aspects of a novel beam-reconfigurable planar series-fed array are addressed to achieve beam steering with frequency tunability over a relatively broad bandwidth. The design is possible thanks to the use of the complementary stripslot, which is an innovative broadly matched microstrip radiator, and the careful selection of the phase shifter parameters.
\end{abstract}

\section{INTRODUCTION}

In order to design a series-fed array with beam reconfiguration over a broad bandwidth, phase shifters can be introduced between two adjacent radiating elements to control the phase and, then, the pointing angle. However, doing that over a broad frequency band requires a non-resonant radiating element. In [1], the authors proposed a radiating element with broad impedance bandwidth, unlike most of the radiators in microstrip technology. This element allowed the design of a novel reconfigurable series-fed array with two functionalities: beam steering and fixed-beam frequency tunability over the LTE band from 1.71 to $2.17 \mathrm{GHz}$ [2]. In this contribution, some aspects of the design of a series-fed array with beam reconfiguration based on a broadband element are addressed.

\section{Design Aspects}

The proposed antenna is shown in Fig. 1, It is a microstrip series-fed array with phase shifters between the radiating elements. The radiator is a complementary strip-slot [1], which consist of a slot etched on the ground plane of a microstrip line and its complementary stub (or strip) superimposed to it in the microstrip layer. The phase shifter is based on a highimpedance microstrip line periodically loaded with lumped elements (varactors), as proposed in [3].

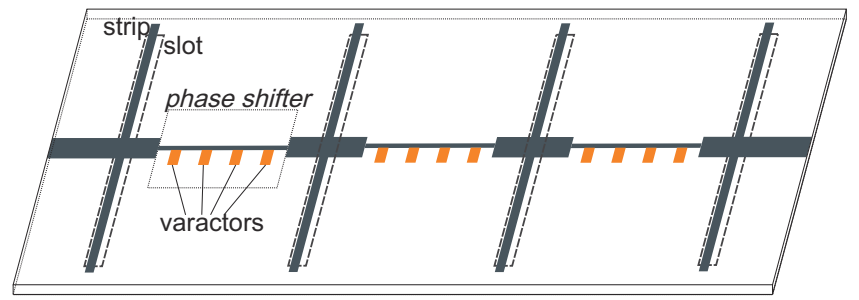

Fig. 1: Layout of the proposed antenna.

\section{A. Effect of the varactor polarization in the distribution of the spatial harmonics}

Although the Floquet's Theorem is stated for infinite periodic structures, it provides accurate information about the radiation frequency bands even for arrays with a few elements. The reverse voltage applied to the varactors modifies the phase shift introduced between the elements and, then, the phase constant of the spatial harmonics when Floquet's Theory is applied. This effect is illustrated in Fig. 2 for a specific geometry (given a distance between elements, a phase shifter and a radiating element). As can be observed, the reverse voltage modifies not only the location of the phase curves with respect to the radiation cone (delimited by the red lines) but also their slope. Therefore, the design must pursue the location of a spatial harmonic inside the radiation cone at each frequency for the design bandwidth, in order to ensure radiation. This is a quite difficult task and does not have a guaranteed solution, because, as the antenna must be electronically reconfigurable, it must be achieved for each reverse voltage. Anyway, the gaps of no radiation, such as the gap between 1.9 and $1.95 \mathrm{GHz}$ in the blue curve of Fig. 2. must be minimised. As the slope of the curves increases, which happens for lower reverse voltages, the appearance of radiation gaps is easier. On the other hand, for low slopes, it can happen that two spatial harmonics are inside the radiation cone at the same frequency and voltage, which would lead to two beams in the radiation pattern. Therefore, there is a trade-off in the phase parameter controlled by the distance between elements, the design of the phase shifter and the geometry of the radiating element. With these three design criteria, unique-beam radiation at all the frequencies and over the maximum range of reverse voltages should be achieved.

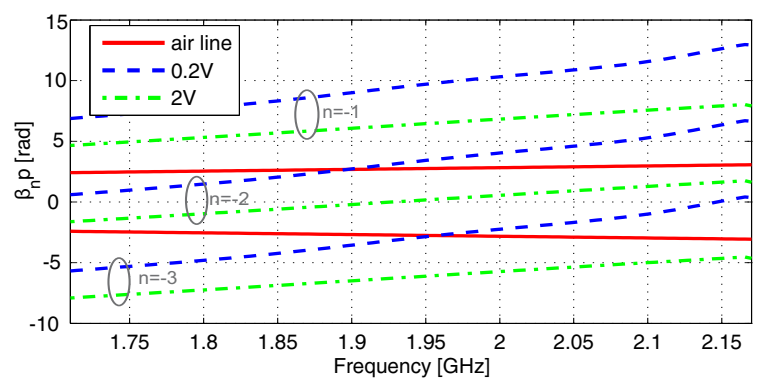

Fig. 2: Spatial harmonics for two different reverse voltages.

B. Influence of the number of stages of the phase shifter on the array performance

In order to study the influence of the number of cells of the phase shifter on the array performance, the total length of the phase shifter is fixed $(55 \mathrm{~mm})$ and the array behaviour 
with a phase shifter of four and five stages is analysed and compared. Fig. 3 show the theoretical pointing angle and the simulated $\left|S_{11}\right|$ versus $V_{R}$ achieved by the array given a phase shifter of four and five stages. As the number of phase shifter stages increases, the phase achieved at a certain frequency with a reverse voltage also increases. This leads to a shift towards right of the $\theta$ versus $V_{R}$ curves and to an increase of the slopes, as Fig. 3b compared to Fig. 3a indicates. Although a higher number of stages might seem beneficial in terms of achieved phase range, attention must be paid to the array losses, not only because an additional stage increases the losses of the phase shifter but also because of the shift of the $\theta$ versus $V_{R}$ curves. $V_{R}$ is inversely proportional to the varactor capacitance and, then, inversely proportional to the varactor losses. As the varactor losses increase with frequency, it is important to obtain radiation with high values of $V_{R}$ at high frequencies and vice versa to minimize losses. In the analysed case, the increase of the number of stages makes necessary low values of $V_{R}$ to get the angles from 135 to $180^{\circ}$ for the highest frequency, which is quite inconvenient. In order to maintain the wide phase range of five stages without compromising the losses, an additional shift of the curves towards right would be advantageous. This can be achieved by adding $50 \Omega$-microstrip line sections to both ends of the phase shifter (offset), thus increasing the distance between elements. This has been done in the analysed case with sections of $5.5 \mathrm{~mm}$, leading to the curves of Fig. 4 (manufactured array).

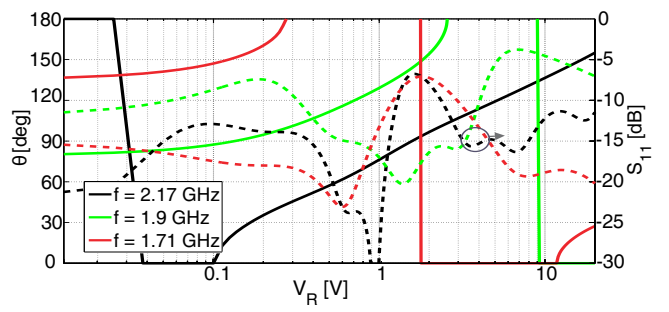

(a) Phase shifter with four stages

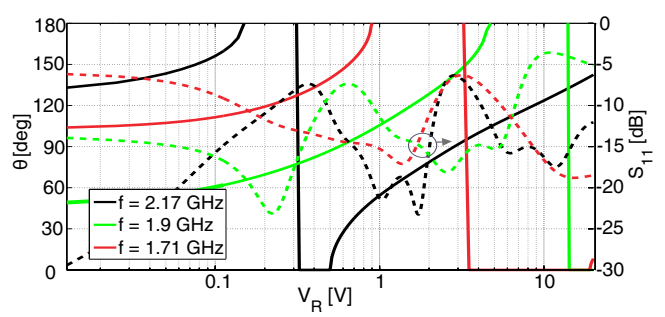

(b) Phase shifter with five stages

Fig. 3: Simulated pointing angle $\theta$ and $\left|S_{11}\right|$ of the array versus the reverse voltage of the varactors.

\section{Results of the Final Design}

A design for the previously-mentioned LTE band with four radiating elements, five stages for the phase shifter and distance between elements of $66 \mathrm{~mm}$ has been manufactured and measured. Good agreement between measurements and simulations is obtained. The array is well-matched $\left(\left|S_{11}\right| \leq\right.$

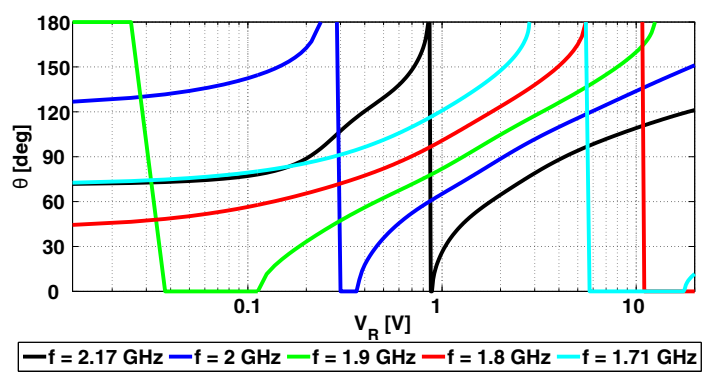

Fig. 4: Phase shifter with five stages and offset (final design).

$-10 d B$ ) for all the reverse voltages except for the broadside region and the no-radiation region. Moreover, the antenna efficiency is high since almost no power reaches the load (a low $\left|S_{21}\right|$ has been obtained).

Fig. 5 illustrates the beam reconfiguration of the antenna. The radiation patterns at two different frequencies (1.9 and $2.05 \mathrm{GHz})$ and for different applied reverse voltages are shown. It is highlighted that the same beam pointing direction can be achieved at different frequencies over the analysed bandwidth by applying the appropriate reverse voltage.

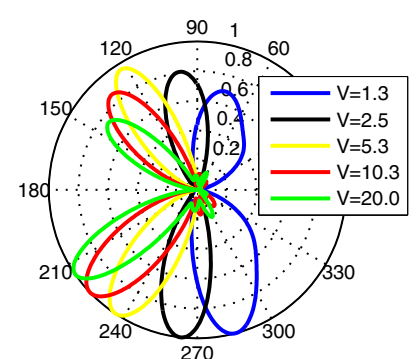

(a) $\mathrm{f}=1.9 \mathrm{GHz}$

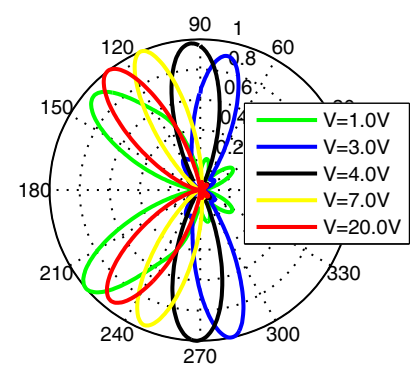

(b) $\mathrm{f}=2.05 \mathrm{GHz}$
Fig. 5: Measured radiation patterns at two different frequencies versus the applied reverse voltage.

\section{CONCLUSIONS}

It has been highlighted that the design of a beamreconfigurable array over broad bandwidth is not straightforward due to the numerous design aspects that influence the final array performance. However, with a broadband radiating element and a careful selection of the number of stages of the phase shifter and distance between elements, it is possible to design an array with beam reconfiguration over a relatively broad frequency band and with significant antenna efficiency.

\section{REFERENCES}

[1] E. Abdo-Sánchez et. al, "Planar Broadband Slot Radiating Element Based on Microstrip-Slot Coupling for Series-fed Arrays," in IEEE Transactions on Antennas and Propagation, vol. 60, no. 12, pp. 6037-6042, Dec. 2012.

[2] E. Abdo-Sánchez et. al, "Electronically Steerable and Fixed-Beam Frequency-Tunable Planar Traveling-Wave Array," accepted for publication in the IEEE Transactions on Antennas and Propagation.

[3] A. Nagra and R. York, "Distributed analog phase shifters with low insertion loss," in IEEE Transactions on Microwave Theory and Techniques, vol. 47, pp. 1705-1711, Sep. 1999. 